“C 2007 IEEE. Personal use of this material is permitted. Permission from IEEE must be obtained for all other uses, in any current or future media, including reprinting/republishing this material for advertising or promotional purposes, creating new collective works, for resale or redistribution to servers or lists, or reuse of any copyrighted component of this work in other works." 


\section{Performance Analysis of Energy Regeneration System of Electric Vehicle with Two Wheels under the Mode of Constant Braking Torque}

\author{
Jiaxin Chen \\ College of Electromechanical \\ Engineering \\ Donghua University \\ Shanghai, China \\ chjiaxin@dhu.edu.cn
}

\author{
Jianguo Thu, Youguang Guo \\ Faculty of Engineering \\ University of Technology, Sydney \\ Sydney, NSW, Australia \\ joe@ieng.uts.edu.au \\ youguang(a)ng.uts.edu au
}

\author{
Jianxun Jin \\ School of Automation Engineering \\ University of Electronic Science and \\ Technology of China \\ Chengdu, Sichuan, China \\ jxinauestc.edu.cn
}

\begin{abstract}
A comprehensive performance analysis is cracial for advance a system to a practical level. This paper presents the performance analysis of energy regeneration system (ERS) of electric vehicle (EV) with two wheels under the model of constant braking torque (CBT). For this, a boost equivalent circuit for main power case (MPC) of ERS is obtaincd firstly, and then a large signal time domain average model of MPC, which constitutes the complete model of ERS along with the other three parts: system dynamics model, permanent de motor driving system model and system performance calculation model, is given here. During modeling ERS, a new method based on state forecast viewer is proposed to identify the state (CCM or DCM) of the MPC. As a nonlinear system, the large signal time domain model of ERS can't be transferred to frequency domain model and implemented in MATLAB/Simulink directly, a method of dividing the system into a slow variable system and a fast variable system is introduced. By running this simulation model, several important performances of ERS are obtained efficiently.
\end{abstract}

\section{INTRODUCTION}

In order to protect the nature surrounding and decrease the danger of using rock oil as a main single energy resource, electric vehicle (EV) has been becoming the main research point in almost developed countries since the decade of 80 in last century ${ }^{[1]}$. As a main vehicle of short distance, the $\mathrm{EV}$ with two wheels has also acquired mud attention in many countries, especially in China and Japan. Although the product of EV with two wheels has entered into the practical market, for being limited by the current technique of storage battery, there are many problems left to be solved till now ${ }^{[2]}$. According to the distributed characteristic of power consumed in EV, the technique of ERS, which can prolong the rated distance by over $20 \%$ in the surrounding of city road, becomes one of important research points at present.

In $\mathrm{EV}$ with two wheels, there are two main kinds of motor: permanent dc motor and brushless dc motor (BLDC). Both of them often have hubcap structures, which will decrease the assembling difficulty and the power consume of dynamics transferring system. For EV with two wheels, the BLDC motor has not advantages than permanent $d c$ motor in totally ${ }^{[3]}$. Many permanent de motor driving systems have been used, and this situation will continue ${ }^{[3]}$. For being the main mode of four ERS modes, the mode of ERS: constant braking torque (CBT) has higher practical value than those others ${ }^{[4]}$. Considering all the above factors, studying ERS of EV with two wheels and dc motor driving system under the mode of CBT is valuable for its application practically. Among many topics, obtaining the effect of ERS on the performance of EV is one of the most important works; it is the base for the application of ERS.

Several papers have done some analysis for $\mathrm{EV}$, however, a systemic performance analysis for $\mathrm{EV}$ is not found till now. For that, a boost equivalent circuit for main power case (MPC) of ERS is obtained, and then the signal average time domain model of the converters is given. Along with the system dynamics model, dc motor driving system and system performance calculation model, the complete average model of ERS is given A new method based on state forecast viewer is proposed to identify the state of MPC. As the nonlinear time domain model can't be transferred to the frequency domain model and implemented in MATLAB/Simulink directly, a method of dividing the system into a fast and slow system is adopted. By running this model, several important performances of ERS are calculated efficiently.

II. DYNAMics Moddel of EV with Two WheELS

According to the theory of mechanical kinematics, the dynamics model of EV with two wheels running in a straight course is shown as follows:

$$
\begin{aligned}
& \left\{\begin{array}{l}
F_{v^{\prime}}=\frac{1}{2} C_{d} A \rho\left(v+v_{w}\right)^{2} \\
F_{f}=m g f \cos a \\
F_{i}=m g \sin a \\
F_{a}=\delta m \frac{d v}{d t}
\end{array}\right. \\
& \delta=1+\frac{J}{m r^{2}} \\
& F_{q}=F_{w^{\prime}}+F_{f}+F_{i}+F_{a}
\end{aligned}
$$

where $v$ is the speed of EV, $v_{w}$ is the windward speed, $J$ is the 
rotational inertia of two wheels, $C_{d}$ is the wind baffle coefficient, $A$ is the windward area, $\rho$ is the air mass density, $r$ is the radius of the wheel, $m$ is the sum of EV mass and burden, $g$ is the gravitation coefficient, $f$ is the roll hinder coefficient, $\delta$ is the equivalent inertia coefficient, $a$ is the gradient angular of road, $F_{q}$ is the propulsive force of motor, and all the left symbols are in accordance with their conventional customs.

\section{MODEZING ENERGY REGENERATION SYSTEM}

\section{A. MPC Equivalent Circuit of ERS}

The typical MPC circuit of permanent dc motor driving system with the function of energy regeneration in EV with two wheels is shown in Fig.1 (a). It is comprised of two controllable switches $T_{1}$ and $T_{2}$ (s.g., MOSFET), two diodes $D_{1}$ and $D_{2}$, a filter capacitor $C$, a permanent dc motor $M_{D}$, and a current sensor $I_{\text {sensor }}$. The switch is turned on and off at the switching frequency $f_{s}=1 / T$ with the ON duty ratio $d_{l}=t_{o n} / T$, where $t_{o n}$ is the interval when the switch is ON. If EV is working in the state of driving, the $T_{2}$ is working in the PWM state, and the $T_{1}$ is turned off all the time. If $\mathrm{EV}$ is working the state of energy regeneration, the $T_{1}$ is working the PWM state, and the $T_{2}$ is turned off all the time. There are two sub-state of energy regeneration: the state of storing the kinetic energy of EV into the inductance of dc motor, and the state of releasing the energy stored in the inductance of dc motor to battery, which are shown in Fig. 1 (b) and Fig.1 (c), correspondingly.

When the EV is working in the state of ERS, the block comprised of $\mathrm{T}_{2}$ and $\mathrm{D}_{2}$ in Fig.1 (b) and Fig.l (c) can be moved to the new position shown in Fig.2 (a). As the $D_{1}$ and $T_{2}$ haven't influences on ERS, both of them are cancelled in Fig.2 (a). By replacing the actual dc motor and other actual components by their corresponding equivalent circuit in Fig.2 (a), then the equivalent circuit with actual components is obtained and shown in Fig.2 (b). The circuit shown in Fig.2 (b) is the typical circuit of the boost converters, where $E_{m}, R_{m}$, and $L_{m}$ are the back emf, the resistance of armature, and the inductance of armature of $\mathrm{dc}$ motor correspondingly; the diode in the ON state is modeled by a constant-voltage battery $V_{2 F}$ and a constant forward resistance $R_{2 O N}$ and in the OFF state by an infinite resistance, all its junction capacitance and lead inductance are omitted; The power MOSFET in the ON state is modeled by a constant resistance $R_{I O N}$ and in the OFF state by an infinite resistance, ant its output capacitance and lead inductance are omitted; the rechargeable battery is modeled by a constant battery $E_{B}$ and a constant resistance $R_{B}$. As all the resistances in Fig. 2 (b) and the $V_{2 F}$ are very small and be omitted, then the equivalent circuit with idea components is obtained and shown in Fig.2 (c). Fig.2 (c) shows the typical boost converters with idea components.

As the output electro-magnetic torque of permanent de motor is proportional to its armature current, the model of CBT can be seen as the model of constant braking current (CBC), and then the typical PI control system of ERS under the model of CBC is shown in Fig.2 (d), where $I_{m}$ is the armature current, $I_{i}$ is the preset current, $\mathrm{d}$ is the turn on ratio cycle of PWM duty cycle, $f_{s}$ is the frequency of sawtooth wave and $m_{c}$ is its slop, $I C$ is a comparator, $P I$ is the controller of proportion and integrator with the saturation limiting output.

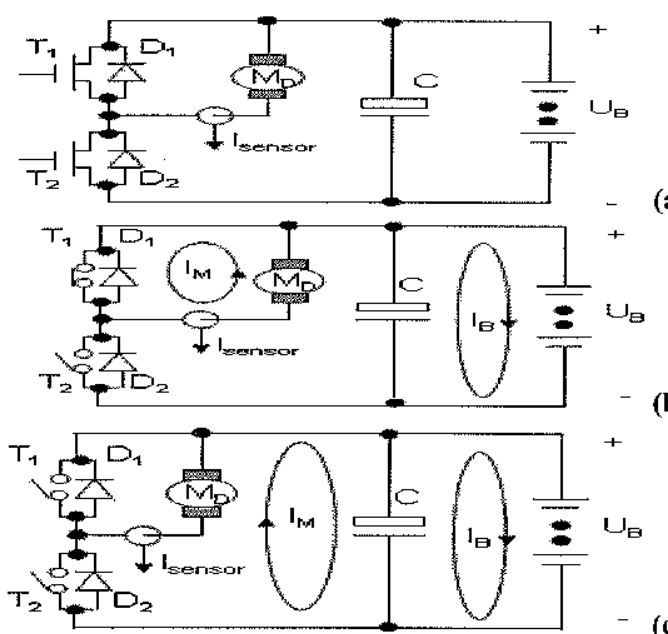

Fig. 1. MPC circuit of ERS based on the permanent dc motor (a) MPC circuit. (b) Storing the kinetic energy in motor. (c) The Releasing the energy stored in motor to battery.
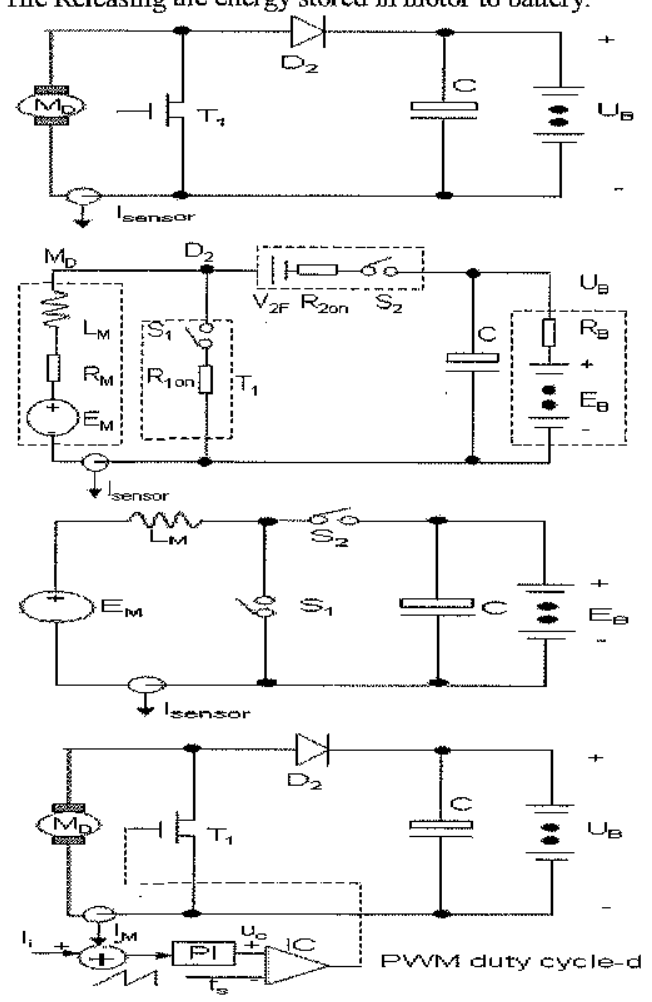

(b)

Fig.2 MPC equivalent circuit of ERS based on the permanent dc motor. (a) Equivalent circuit with actual components. (b) Equivalent circuit with actual equivalent components. (c) Equivalent circuit with idea equivalent components. (d) PI control system of ERS under the model of CBC. 


\section{B. Large Signal Time Domain Average Model}

The model of ERS is comprised of three parts: the signal average model of boost converter, the system dynamics model, the model of permanent dc motor and system performance calculation model. According to Fig.2 (c) and (d), the inductance $L_{m}$ currents can follow in continuous conduction mode (CCM) or in discontinuous conduction mode (DCM). The typical inductor current waveform of DCM is shown in Fig.3, where $d_{1}, d_{2}$ and $d_{3}$ are ratio cycle of PWM duty cycle, correspondingly. In CCM, as the $d_{3}=0, C C M$ can be seen as a special condition of DCM.

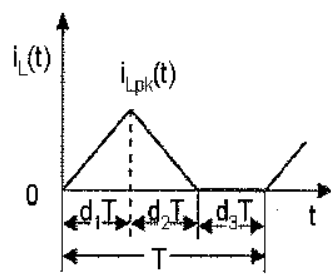

Fig. 3 Typical inductor current waveform of DCM

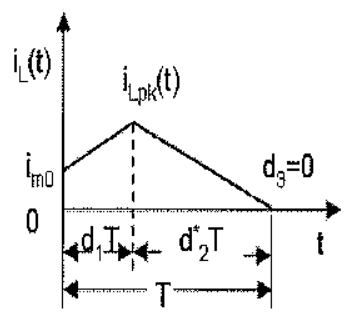

Fig.4 General critical mode
The signal average model of boost converter PI controlled can be obtained by following steps:

$$
\left\{\begin{array}{l}
\frac{d i_{m}}{d t}=\frac{1}{L_{m}}\left[E_{m} * d_{1}+\left(E_{m}-E_{B}\right) * d 2+0 * d 3\right] \\
d_{1}=\frac{U_{c}}{m_{c}} f_{s} \\
U_{c}=K_{p} e(t)+K_{i} \int e(t) d t \\
e(t)=i_{i}-i_{m}
\end{array}\right.
$$

where $K_{p}$ and $K_{i}$ are the coefficients of proportion and integrated correspondingly.

As the equations for calculating the value of $d_{2}$ and $d_{3}$ in the mode of CCM and DCM are different, a method of identifying the operation mode by using the past average current of inductance was introduced in paper [5]. Its process of identifying the operation mode as follows:

$$
d_{2}^{*}=\frac{2 * I_{m} * f_{s}^{*} L_{m}}{E_{m} * d_{1}}-d_{1}
$$

where $I_{m}$ is the average current of inductance $L_{m}$

If $d_{2}{ }^{*}>=1-\mathrm{d}_{1}$, operation in CCM; If $\mathrm{d}_{2}{ }^{*}<1-\mathrm{d}_{1}$, operation in DCM. In CCM and DCM, $\mathrm{d}_{2}$ and $\mathrm{d}_{3}$ are decided by the equations of (6) and (7), correspondingly.

$$
\begin{aligned}
& \left\{\begin{array}{l}
\mathrm{d}_{2}=1-d_{1} ; \\
d_{3}=0 ;
\end{array}\right. \\
& \text { And } \\
& \left\{\begin{array}{l}
\mathrm{d}_{2}=d_{2}^{*} ; \\
d_{3}=1-d_{1}-d_{2} ;
\end{array}\right.
\end{aligned}
$$

It is obvious to find that the method in paper [5] will delay system analysis for one cycle; this may cause unbeknown question. Considering on that fault, a method based on identifying current of inductance at the start up of each cycle is introduced in paper [6]. As the current state of inductance at start up can't decide the operation mode (CCM or DCM), there still exists disparity. Further more, the equation (5) is also used in paper [6] and the $I_{m}$ is the average current of inductance $L_{m}$ in past time, the delay will also happen; this method can't solve the delay question. Here a new method is introduced.

Supposing that system is operating in critical mode during one cycle (shown in Fig.4), and the start current of inductance in each cycle is $i_{m o}$, then the duty cycle $d_{2}{ }^{*}$ can be obtained at the start up of each cycle by following equation ahead:

$$
i_{m 0}+\frac{E_{m} * d_{1}}{L_{m}} * T=\frac{E_{B}-E_{n 1}}{L_{m}} * d_{2}^{*} * T .
$$

Then,

$$
d_{2}^{*}=\frac{i_{m 0} * f_{s} * L_{m}+E_{m} * d_{1}}{E_{B}-E_{m}} .
$$

Then, if $d_{2}{ }^{*}>=1-\mathrm{d}_{1}$, operation in CCM; If $\mathrm{d}_{2}{ }^{*}<1-\mathrm{d}_{1}$, operation in DCM. In CCM and DCM, $d_{2}$ and $d_{3}$ are still decided by the equations (6)-(7), correspondingly. As the Equation (8) can identify the operation mode at start up by using current data, it constitutes a state forecast viewer and has solved the above delay question. As the process of calculating the average current of $L_{m}$ is omitted in the new method, it is helpful to increase the efficiency of simulation. The signal average time domain model of PWM boost converters is comprised of equations (4), (6)-(8)

The model of permanent dc motor driving system is shown in the equations (9).

$$
\left\{\begin{array}{l}
E_{m}=K_{e} * \omega \\
T_{c}=K_{T} * i_{m} \\
F_{q}=\left(T_{e}-\sigma_{0} \omega\right) / r \\
v=\omega * r
\end{array}\right.
$$

where $K_{e}$ is the coefficient of back emf, $K_{T}$ is the coefficient of electro-magnetic output torque of motor, $\omega$ is the angular velocity of motor, $\sigma_{0}$ is the damp coefficient of motor. As the motor with hubcap structures, both the transferring radio and transferring efficiency are 1.

Based on this model of equations (1)-(4) and (6)-(9), several performances of the CBT can be obtained. During the vehicle run from the start speed $v_{0}$ to the speed $v_{E}$, the power of energy regeneration $P_{r}$ is calculated by

$$
P_{r}=E_{B} * I_{n} * d_{2}
$$

The braking distance $S_{B r a k e}$ and the efficiency of energy regeneration $\eta_{B r a k e}$ are calculated by equations of (10) and (11).

$$
\begin{aligned}
& S_{\text {Brake }}=\int_{0}^{t_{E}} v d t, \quad ; v(0)=v_{0}, v\left(t_{E}\right)=v_{E} \\
& \eta_{\text {Brake }}=\frac{\int_{0}^{t_{E}} p_{r} d t}{0.5 \sigma m\left(v_{0}{ }^{2}-v_{E}{ }^{2}\right)} .
\end{aligned}
$$

All the equations (1)-(4) and (6)-(12) constitute the complete large signal average time domain model of EV.

\section{IMPLEMENT IN MATALB/ SIMULINK}

Comparing with the simulation with actual components, the signal average method can transform some nonlinear systems to linear systems which can run faster. From the complete large signal average time domain model of EV (cquations (1)-(12)), 
the ERS of EV with two wheels under the model of CBC is still nonlinear; This nonlinear characteristic causes the question that the signal average time domain model can't be transferred to the system transferring function model and implemented in MATLAB/Simulink surrounding directly. In order to take more advantages from the signal average method as possible as it can, some simply process is being proceed in implementing the signal average time domain model in Matalb/Simulink surrounding. Firstly, considering on $10 \mathrm{kHz}$ frequency of the PWM, the system dynamics model varies in rather lower speed, then the back emf $-E_{m}$ of dc motor can be seen as a constant variable during one PWM cycle, and then the large signal average model of boost converters (CCM or DCM) can also be seen as a linear model. Secondly, in system dynamics model, only the wind baffle equation is nonlinear. As it has definite analytical equation and varies in rather lower speed, it can be solved by the analytical method in lower frequency. All left model can be transferred to frequency domain model and can be implement in MATLAB/Simulink surrounding directly. This divide the system into two parts: one varies faster, and the other

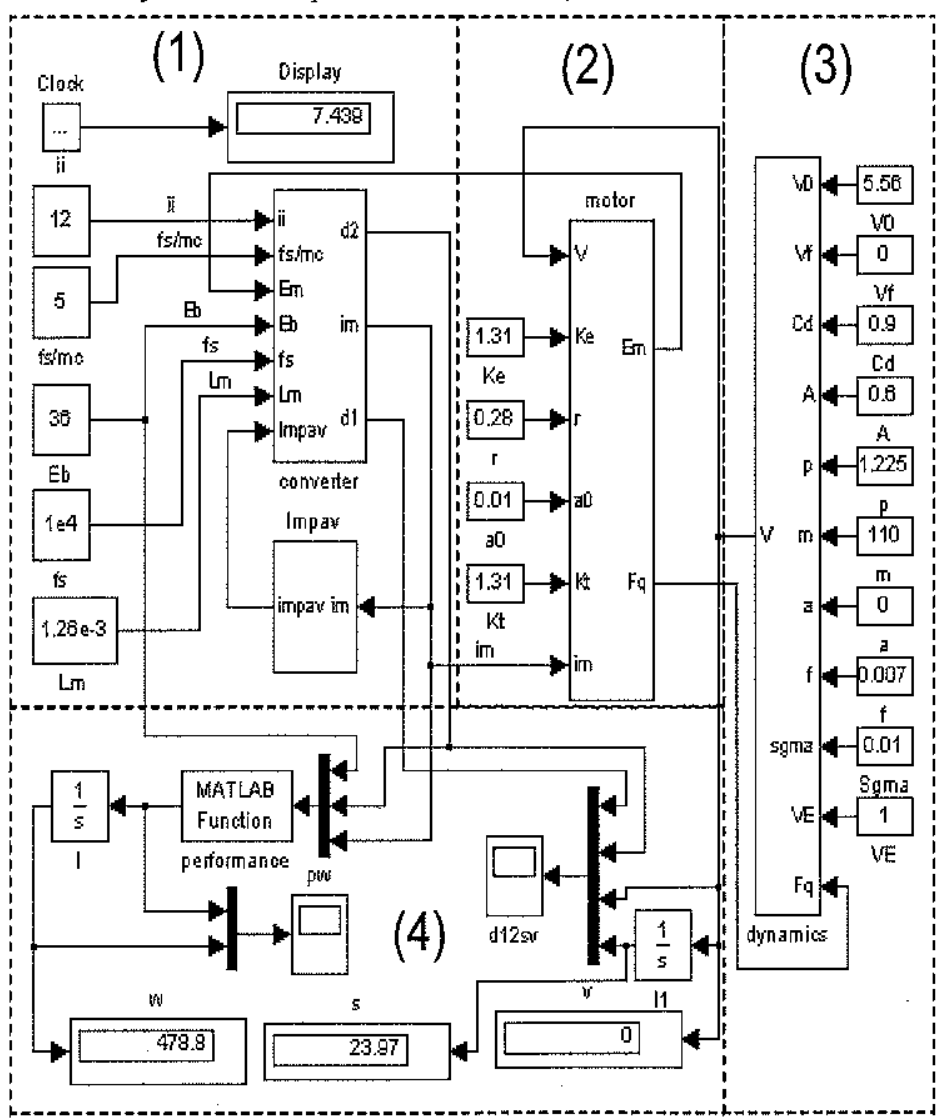

Fig. 5 The complete simulation model based on Matlab/Simulink: (1) ERS model; (2) dc motor driving system model; (3) system dynamics model; (4) performance analysis model. varies slower. This fast and low system simulation model in MATLAB/Simulink surrounding can be obtained and shown in Fig. 5. Fig. 6 is the detail structure of ERS simulation model. The parameters for simulation as follows:

$K_{e}=K_{T}=1.31, r=0.28 \mathrm{~m}, v_{0}=20 \mathrm{~km} / \mathrm{h}, v_{e}=3.6 \mathrm{~km} / \mathrm{h}, f_{\mathrm{s}}=10 \mathrm{KHz}$, $m_{c}=5 e 4, E_{b}=36 v, L_{m}=1.26 \mathrm{mh}, v_{f}=0, a=0, m=110 \mathrm{~kg}, f=0.007$, $A=0.6 \mathrm{~m}^{2}, C_{d}=0.9, \rho=1.225 \mathrm{~kg} / \mathrm{m} . \delta=1.05, \sigma_{0}=0.015$.

By inputting all above data to simulation model and running, several important performances such as the braking distance, the braking time, the efficiency and the sum of energy regeneration system can be obtained automatically. Further more, the braking distance-S, the energy regeneration efficiency-eff and braking time-t from the $20 \mathrm{~km} / \mathrm{h}-3.6 \mathrm{~km} / \mathrm{h}$ under different braking current-li can also be calculated. The simulation results are shown in Fig. 6 and Fig.7. From the simulation results, it is easy to find that the efficiency of ERS is larger than $21.6 \%$, and the speed of EV, the duty cycle $d_{2}$ will tends to smaller while ERS works continuously, all these phenomena are in accordance with analysis result theoretically.

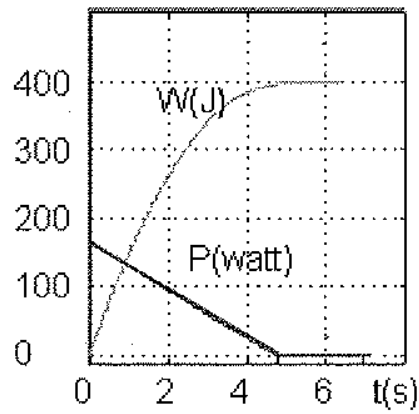

(a)

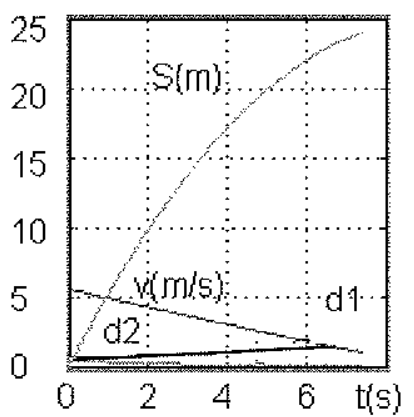

(b)

Fig.7 Simulation results under the constant braking current-12A: (a) regeneration energy $-\mathrm{W}$ and regeneration power- $\mathrm{P}$ vs braking time-t; (b) the braking distance-S, the speed during braking- $\mathrm{V}$, the duty cycle-dl and $\mathrm{d} 2$ vs braking time-t. 


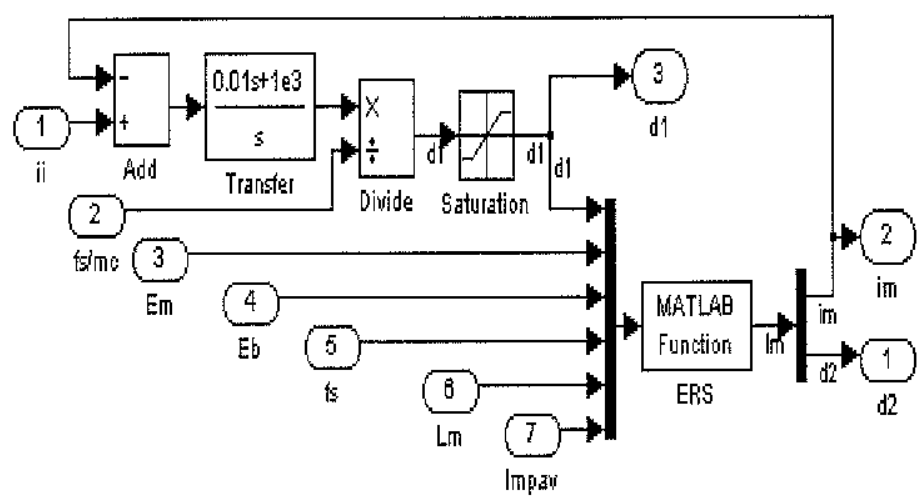

Fig. 6 the detail structure of ERS simulation model

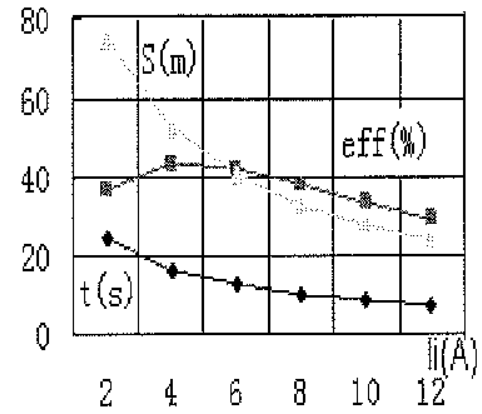

Fig. 8 Performance analysis: the braking distance-S, the energy regeneration efficiency-eff and braking time-t from the $20 \mathrm{~km} / \mathrm{h}-3.6 \mathrm{~km} / \mathrm{h}$ under different braking current-Ii.

\section{CONCLUSIONS}

This paper has presented the performance analysis of energy regeneration system (ERS) of electric vehicle (EV) with two wheels under the model of constant braking torque (CBT). A boost equivalent circuit for main power case (MPC) of ERS, the complete model of ERS and its simulation model are obtained. Both the new method based on state forecast viewer for identifying the state (CCM or DCM) of the MPC and the method based on different sampling period for implementing nonlinear system into MATLAB/Simulink surrounding are proposed. The model has proved to be efficient, by running this model, several important performances of ERS for the application in designing ERS are calculated automatically.

\section{REFERENCES}

[1] JX Chen "The Solve Way of Some Problems in the Control of Electric Scooter. "Electric Motor and Electric Apparatus, China, pp. $38-42$, May 2001

[2] J.X.Chen, "The Mathematics Model of Electric Vehicle Rating Distance and Its Application." Electric Drive Automation, China, Vol. $2 \neq$,pp. 55-59, April 2002

[3] JX Chen "The Compare of motor and the Drive Methods Used in Electric Vehicle." Electric Motor and Electric Apparatus, China, pp. 16-18, January 2002

[4] J.X.Chen, H.Cai and M.S.Sun, "The research of energy regencration system and its reliability in Electric Vehicle" PEDS 2003 :IEEE, The FIFTH INTERNATIONAL CONFERENCE ON POWER ELECTRONICS AND DRIVE SYSTEMS, VOLS 1 AND 2, PROCEEDINGS : $86+-867,2003$

[5] B.Yaakovs, "Average simulation of PWM converters by direct implement of behaviour relationships, ${ }^{n}$ PToc of IEEE APEC[C] Piscatiaway USA: IEE Inc,pp.S10-5I6,1993

[6] S.Z.Pan,Z.M.Qian, N.Lei,"A novel average model for unified forward dc-dc converter," Proceedings of the CSEE,China, vol, 21 pp.58-62, June 200 